\title{
Non-Franck-Condon Effects Induced by Orbital Evolution and Cooper Minima in Excited-State Photoionization of $\mathrm{OH}$
}

\author{
J. A. Stephens and V. McKoy \\ Arthur Amos Noyes Laboratory of Chemical Physics, California Institute of Technology, Pasadena, California 91125 \\ (Received 22 August 1988)
}

\begin{abstract}
We show that strong non-Franck-Condon effects can arise from photoionization of molecular Rydberg orbitals which evolve rapidly with internuclear distance and display Cooper minima in their photoelectron spectrum. The non-Franck-Condon mechanism should be most pronounced in excited-state photoionization of diatomic hydrides. The mechanism is illustrated with specific ab initio predictions of ion vibrational distributions for a proposed $(3+1)$ one-color or $(2+1)$ two-color resonant multiphoton ionization measurement of $\mathrm{OH}$ via the $D^{2} \Sigma^{-}\left(1 \pi^{2} 5 \sigma\right)$ state
\end{abstract}

PACS numbers: $33.80 . \mathrm{Rv}, 33.90 .+\mathrm{h}$

The photoionization of molecular Rydberg states is generally expected to occur with the preservation of vibrational quantum numbers. ${ }^{1-3}$ For Rydberg states which are well described by a single, highly excited electron with a specific ionic core, the near identity of the neutral and ionic potential surfaces leads to FranckCondon distributions, i.e., $\Delta v=v^{+}-v^{\prime}=0$. Here $v^{\prime}$ and $v^{+}$are vibrational quantum numbers for the neutral Rydberg state and the ion, respectively. Important exceptions to this result have recently been shown to arise from electronic autoionization of repulsive doubly excited states ${ }^{3-5}$ in photoionization of the $C^{1} \Pi_{u}$ Rydberg state $\mathrm{H}_{2}$, and shape-resonance-induced non-FranckCondon effects ${ }^{6-9}$ in photoionization of the $C^{3} \Pi_{g}$ Rydberg state of $\mathrm{O}_{2}$. These effects derive from considerations of final-state photoionization dynamics. In this Letter, we predict a mechanism for producing significant non-Franck-Condon vibrational distributions which derives solely from properties of the initial Rydberg orbital. These properties are the following: (i) the Rydberg level must have at least one radial node in its wave function, so that a Cooper minimum ${ }^{10,11}$ may occur in some portion of the photoionization spectrum, and (ii) the Rydberg orbital should evolve rather rapidly into its united or separated atom limits, over a range of internuclear distance associated with low-vibrational levels, e.g., $v^{\prime} \sim 0-3$. Molecular Rydberg orbitals with these characteristics typically occur in diatomic hydrides, an important class of species in multiphoton ionization, photodissociation, and photofragmentation problems. Chupka ${ }^{3}$ has pointed out the likely significance of Cooper minima in excited-state molecular photoionization. However, experimental and theoretical information was insufficient then to identify specific mechanisms which could result in anomalous ion vibrational distributions. These ideas are now discussed specifically in terms of electronic structure and $a b$ initio calculations of vibrationally resolved photoionization spectra of the $D^{2} \Sigma^{-}\left(1 \pi^{2} 5 \sigma\right)$ Rydberg state of $\mathrm{OH}$.

In Fig. 1 we show potential-energy curves for the
$X^{2} \Pi\left(1 \pi^{3}\right)$ ground state of $\mathrm{OH}$, the $X^{3} \Sigma^{-}\left(1 \pi^{2}\right)$ state of $\mathrm{OH}^{+}$, and a set of excited-state curves leading to the ion with the electronic configuration ${ }^{2} \Sigma^{-}\left(1 \pi^{2} n \sigma\right)$, where $n=4-8 .{ }^{12,13}$ Following Mulliken, ${ }^{16}$ we have correlated the states of neutral $\mathrm{OH}$ with states of the united atom fluorine, ${ }^{15,17}$ and have also indicated some states of the separated atoms. The repulsive nature of the ${ }^{2} \Sigma^{-}\left(1 \pi^{2} 4 \sigma\right)$ state arises from "Rydbergization" of an antibonding $2 p_{\mathrm{O}}-1 s_{\mathrm{H}}$ molecular orbital into the fluorine $3 s$ orbital at small $R$. $^{18-20}$

We have performed (Hartree-Fock) improved-virtualorbital $^{21}$ calculations of the ${ }^{2} \Sigma^{-}\left(1 \pi^{2} 5 \sigma\right)$ and ${ }^{2} \Sigma^{-}\left(1 \pi^{2} 6 \sigma\right)$ Rydberg states, in which the excited orbital has been optimized in the field of the $X^{3} \Sigma^{-}\left(1 \pi^{2}\right)$ ion. In Table I we give total electronic energies at five inter-

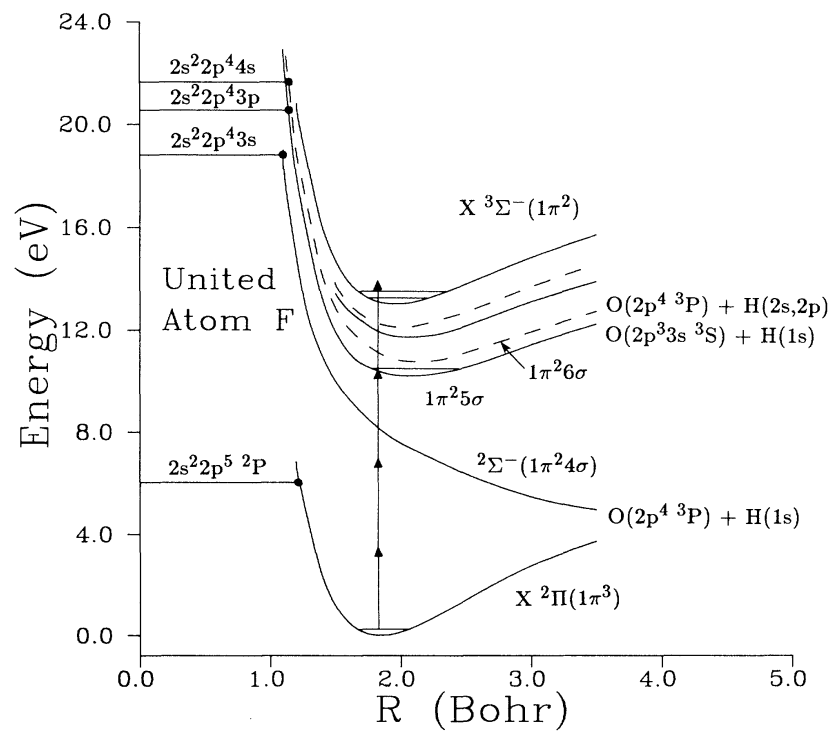

FIG. 1. Potential-energy curves for the ground and first ionic state of $\mathrm{OH}$, and the excited states of the electronic configuration ${ }^{2} \Sigma^{-}\left(1 \pi^{2} n \sigma\right)$ (from Refs. 12-14). The atomic energy levels are from Ref. 15. 
TABLE I. Total energies of $\mathrm{OH}^{3} \Sigma^{-}$Rydberg states and principal angular momentum composition of $n \sigma$ Rydberg orbitals. $^{\mathrm{a}}$

\begin{tabular}{ccccccc}
\hline \hline & \multicolumn{3}{c}{$1 \pi^{2} 5 \sigma$} & \multicolumn{2}{c}{$1 \pi^{2} 6 \sigma$} \\
$R^{\mathrm{b}}$ & $-E_{\text {total }}^{\mathrm{b}}$ & $s(\%)$ & $p(\%)$ & $-E_{\text {total }}$ & $s(\%)$ & $p(\%)$ \\
\hline 1.200 & 74.8138 & 12.1 & 87.8 & 74.7804 & 86.9 & 13.1 \\
1.834 & 75.0868 & 22.1 & 77.1 & 75.0588 & 75.6 & 23.0 \\
2.043 & 75.0848 & 34.9 & 63.3 & 75.0619 & 54.3 & 42.7 \\
2.250 & 75.0735 & 57.8 & 38.1 & 75.0544 & 35.6 & 59.3 \\
3.000 & 75.0164 & 86.4 & 5.3 & 74.9930 & 8.5 & 90.9 \\
\hline \hline
\end{tabular}

${ }^{a}$ The calculated vertical excitation energy of the ${ }^{2} \Sigma^{-}\left(1 \pi^{2} 5 \sigma\right)$ state from the $X^{2} \Pi\left(1 \pi^{3}\right)$ ground state at $R_{e}=1.8342 a_{0}$ is $11.3 \mathrm{eV}$. The experimental value is $10.2 \mathrm{eV}$ (Ref. 22).

${ }^{\mathrm{b}}$ Internuclear distances and total energies are in atomic units.

nuclear distances and angular momentum compositions of the $5 \sigma$ and $6 \sigma$ orbitals, as determined by single-center expansion of the wave function about the center of mass. The contributions to the $5 \sigma$ and $6 \sigma$ orbitals for $l \geq 2$ are essentially negligible, and are not included in Table I. Treating the Rydberg nature of these orbitals explicitly by the improved-virtual-orbital method results in quite accurate reproduction of level splittings. The calculated quantum defect of the $5 \sigma$ orbital at $R_{e}=2.043 a_{0}$ is 0.723 , and is the first member of a " $p$ " Rydberg series converging to the ionization threshold at $13.01 \mathrm{eV} .^{22,23}$ The $6 \sigma$ orbital has a quantum defect of 1.391 , and is the first member of an " $s$ " series whose potential-energy curve resembles the ion. The $7 \sigma$ and $8 \sigma$ orbitals are the second members of the $p$ and $s$ Rydberg series, shown respectively as the unlabeled solid and dashed curves in Fig. 1. Although these orbitals correlate with the united atom $3 p$ and $4 s$ orbitals, the nonspherical molecular field at larger $R$ results in significant admixture of the $s$ - and $p$-wave components. As seen in Table $\mathrm{I}$, the angular momentum composition varies rapidly as the internuclear distance changes adiabatically, with limits restricted by symmetry and energetic requirements at small and large $R$. Accompanying this rapid variation in angular momentum composition are changes in nodal structure of the excited-state wave function. This has important consequences for photoionization from the molecular excited state.

We have studied photoionization of the ${ }^{2} \Sigma^{-}\left(1 \pi^{2} 5 \sigma\right)$ Rydberg state using frozen-core, numerical HartreeFock continuum orbitals. These orbitals were obtained using the iterative Schwinger variational method, ${ }^{24,25}$ and employed the multiplet-specific Hartree-Fock potential used in calculations of the photoionization cross section for producing $X^{3} \Sigma^{-}\left(1 \pi^{2}\right)$ ions from the $\mathrm{OH}$ ground state. ${ }^{26}$ To account for non-Franck-Condon effects, we included full kinetic energy and $R$ dependence in the bound-free transition moment

$$
\bar{r}_{f v^{+} i v^{\prime}}=\int d R \chi_{v^{+}}^{*}(R) r_{f i}(k ; R) \chi_{v^{\prime}}(R) .
$$



FIG. 2. Calculated (velocity form) photoionization cross sections for the $5 \sigma \rightarrow k \pi$ channel at various internuclear distances. The cross sections assume an ionization potential of $2.81 \mathrm{eV}$

Here, $r_{f i}(k ; R)$ is the transition moment calculated at fixed internuclear distance $R$ and electron momentum $k$, for a bound (Rydberg) initial state $|i\rangle$ and continuum (photoelectron +ionic core) final state $|f\rangle$. The vibrational wave functions for the ${ }^{2} \Sigma^{-}\left(1 \pi^{2} 5 \sigma\right)$ Rydberg state and the ${ }^{3} \Sigma^{-}\left(1 \pi^{2}\right)$ ion are indicated by $\chi_{v^{\prime}}$ and $\chi_{v}+$, respectively. The Rydberg initial state and photoelectron orbitals for the $5 \sigma \rightarrow k \sigma$ and $5 \sigma \rightarrow k \pi$ continuum channels were obtained over a range of internuclear distance which spans the $v^{+}=0-5$ vibrational levels of the ion. The vibrational wave functions for the Rydberg and ionic states were obtained by numerical integration of the potential curves of van Dishoeck and co-workers ${ }^{12,13}$ and Werner, Rosmus, and Reinsch, ${ }^{14}$ respectively.

In Fig. 2 we show photoionization cross sections for the $5 \sigma \rightarrow k \pi$ channel at several values of the internuclear distance. The principal result is demonstration that the electronic transition moment (cross section) is a strong function of internuclear distance, and hence the usual Franck-Condon factorization of $\bar{r}_{f_{v}}{ }_{i i^{\prime}}$ becomes invalid. As a consequence, we can expect significant departures from the $\Delta v=0$ propensity rule for producing molecular ions in different vibrational states (see Fig. 4). Prior to focusing on this, we summarize the essential features of these fixed-nuclei cross sections, which underlie the predicted non-Franck-Condon behavior.

At values of $R \lesssim 2.1 a_{0}$, the cross section in Fig. 2 is markedly diminished $\sim 5-\mathrm{eV}$ photon energy, which results from canceling contributions to the photoionization matrix elements. The cancellation occurs mainly in the $5 \sigma \rightarrow k \pi(l=2)$ channel, and results from a single radial 


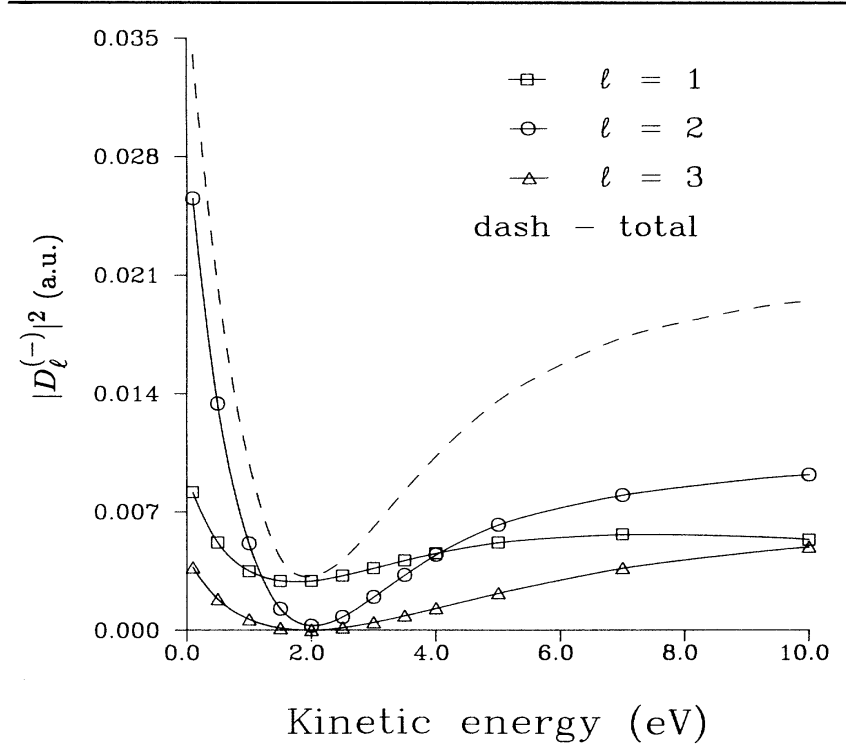

FIG. 3. Calculated dipole strengths (incoming-wave normalized, velocity form) for the $5 \sigma \rightarrow k \pi$ channel, $l=1-3$ continuum partial waves, at $R=1.8342 a_{0}$.

node in the $p$-wave $(l=1)$ component of the $5 \sigma$ orbital. In Fig. 3 we show the (incoming-wave normalized) partial-wave dipole strengths $\left|D_{l}^{(-)}\right|^{2}$ for the $5 \sigma \rightarrow k \pi$ channel at $R=1.8342 a_{0}$. The continuum $d$-wave $(l=2)$ component determines the overall spectral behavior, which exhibits a zero at $\sim 2-\mathrm{eV}$ kinetic energy. As seen in Table I, the $5 \sigma$ orbital is predominantly $p$ wave (i.e., " $3 p \sigma$," correlating to the $3 p$ orbital of fluorine), whose nodal structure results in the Cooper minimum. This one-electron feature is well-documented in ground ${ }^{10,11}$ and excited-state ${ }^{27}$ photoionization of atoms, and the ground state of molecules with "lone-pair" molecular orbitals. ${ }^{28,29}$ As the internuclear distance increases beyond $R \sim 2.1 a_{0}$, the $5 \sigma$ orbital rapidly evolves into one with predominantly excited $s$-wave $(l=0)$ character. Here the $5 \sigma$ orbital overlaps more effectively with the $k \pi$ $(l=1)$ continuum orbital at these photoelectron energies, and as the photon energy increases, the cross section exhibits a rapid and monotonic decrease. This is similar to that predicted for photoionization from excited $s$ orbitals of atoms. ${ }^{30}$ The $5 \sigma \rightarrow k \sigma$ cross sections (not shown) are considerably less $R$ dependent, and do not show a minimum as striking as in the $5 \sigma \rightarrow k \pi$ channel.

In Fig. 4 we show calculated ion vibrational distributions for photoionization of the $v^{\prime}=0-3$ levels of the ${ }^{2} \Sigma^{-}\left(1 \pi^{2} 5 \sigma\right)$ Rydberg state. The photon energies for each frame correspond to the one-photon ionization step of a $(3+1)$ one-color, resonant-enhanced multiphoton ionization experiment ${ }^{1,2}$ (indicated in Fig. 1). For a single-color experiment this scheme seems advantageous, since the $a^{1} \Delta$ ionic state ${ }^{23,26}\left(V_{\text {I.P. }}=15.17 \mathrm{eV}\right)$ is energetically inaccessible, and possible effects due to channel coupling would be avoided. A $(2+1)$ two-color experi-
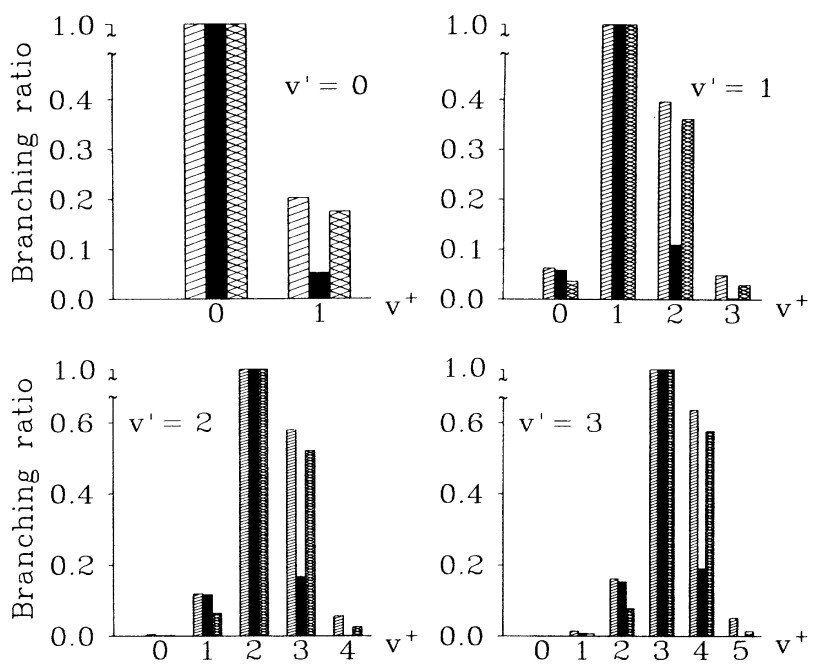

FIG. 4. Calculated vibrational branching ratios for $\mathrm{OH}$ $D^{2} \Sigma^{-}\left(v^{\prime}=0-3\right)$ photoionization. The one-photon energies for the $v^{\prime}=0-3$ frames, respectively, are $3.42,3.52,3.62$, and 3.73 $\mathrm{eV}$. In each frame the ratios were obtained by dividing the $\Delta v=0$ absolute intensity. Franck-Condon ratio (solid bar); full, length form (crossed bar); full, velocity form (crosshatched bar).

ment could probe the same photoelectron energies, however. The ratio of Franck-Condon factors $\left|\left\langle v^{+} \mid v^{\prime}\right\rangle\right|^{2}$ is at most $\sim 0.2$ (e.g., the $v^{\prime}=3 \rightarrow v^{+}=4$ transition). By inclusion of the $R$ dependence of the photoionization matrix elements exactly, we find very large deviations from Franck-Condon predictions. For the $\Delta v=v^{+}-v^{\prime}=1$ branching ratios, the enhancement over the FranckCondon results are typically $\sim 3$. For the $\Delta v=2$ transitions, the enhancements range from factors of $\sim 10$ to 50. These enhancements in the vibrational branching ratios are comparable to those induced, e.g., by electronic autoionization $^{2-5}$ of doubly excited states, in photoionization of the $C^{1} \Pi_{u}$ state of $\mathrm{H}_{2}$.

In summary, we have discussed a new mechanism by which molecular ion vibrational distributions produced in resonant-enhanced multiphoton ionization experiments can substantially deviate from the well-known $\Delta v=0$ propensity rule. We have predicted the presence of a Cooper minimum in the photoionization spectrum of the $D^{2} \Sigma^{-}\left(1 \pi^{2} 5 \sigma\right)$ Rydberg state of $\mathrm{OH}$, and have shown how the evolution of this feature with changes in internuclear distance mediates the non-Franck-Condon behavior. Such behavior should be clearly observable in measured vibrational branching ratios. Finally, we believe that these considerations will not be restricted to the present $\mathrm{OH}$ example, but will generally be most pronounced in diatomic hydrides, since their electronic structure is close to the corresponding united atom.

The authors acknowledge helpful discussions with Dr. S. N. Dixit at an early stage of this work. This work was 
supported by the National Science Foundation (Grant No. CHE-8521391), Air Force Office of Scientific Research (Contract No. 87-0039), and the Office of Health and Environmental Research of the U.S. Department of Energy (Grant No. DE-FG03-87ER60513). We also acknowledge use of resources of the San Diego Supercomputer Center, which is supported by the $\mathrm{Na}$ tional Science Foundation.

${ }^{1}$ R. N. Compton and J. C. Miller, in Laser Applications in Physical Chemistry, edited by D. K. Evans (Dekker, New York, 1988), Sec. 4.

${ }^{2}$ P. M. Dehmer, J. L. Dehmer, and S. T. Pratt, Comments At. Mol. Phys. 19, 205 (1987), Sec. 3.

${ }^{3}$ W. A. Chupka, J. Chem. Phys. 87, 1488 (1987).

${ }^{4}$ A. P. Hickman, Phys. Rev. Lett. 59, 1553 (1987).

${ }^{5}$ S. N. Dixit, D. L. Lynch, V. McKoy, and A. U. Hazi (to be published)

${ }^{6}$ P. J. Miller, L. Li, W. A. Chupka, and S. D. Colson, J. Chem. Phys. 89, 3921 (1988).

${ }^{7}$ J. A. Stephens, M. Braunstein, and V. McKoy, J. Chem. Phys. 89, 3923 (1988).

${ }^{8}$ P. J. Miller, W. A. Chupka, J. Winniczek, and M. G. White, J. Chem. Phys. 89, 4058 (1988).

${ }^{9}$ M. Braunstein, J. A. Stephens, and V. McKoy, J. Chem. Phys. (to be published).

${ }^{10}$ U. Fano and J. W. Cooper, Rev. Mod. Phys. 40, 441 (1968), Sec. 4.5.

${ }^{11}$ S. T. Manson, Phys. Rev. A 31, 3698 (1985), and references quoted therein.

${ }^{12}$ E. F. van Dishoeck, S. R. Langhoff, and A. Dalgarno, J. Chem. Phys. 78, 4552 (1983).

${ }^{13}$ E. F. van Dishoeck and A. Dalgarno, J. Chem. Phys. 79, 873 (1983).

${ }^{14}$ H.-J. Werner, P. Rosmus, and E.-A. Reinsch, J. Chem. Phys. 79, 905 (1983).

${ }^{15}$ C. E. Moore, Atomic Energy Levels, as Derived from the
Analyses of Optical Spectra (U.S. GPO, Washington, DC, 1971), Vol. 1, pp. 60-61.

${ }^{16}$ R. S. Mulliken, Rev. Mod. Phys. 4, 1 (1932).

${ }^{17}$ Using data from Ref. 15, the Rydberg atomic energy levels in Fig. 1 were obtained by averaging over the many allowed $L S$ multiplets with the configurations $2 s^{2} 2 p^{4} 3 s$ and $2 s^{2} 2 p^{4} 3 p$, and hence are not labeled by term symbols. The energy of the $2 s^{2} 2 p^{52} P$ fluorine ground state has been set at the dissociation limit of the OH $A^{2} \Sigma^{+}\left(3 \sigma 1 \pi^{4}\right)$ state (Ref. 12). The spindoublet $3 \sigma 1 \pi^{4}$ configuration is the highest-energy configuration which correlates to atomic $2 p^{5}$ configuration.

${ }^{18} \mathrm{H}$. Lefebvre-Brion and R. W. Field, Perturbations in the Spectra of Diatomic Molecules (Academic, Orlando, 1986), Chap. 4, Sec. 2.

${ }^{19}$ R. S. Mulliken, Acct. Chem. Res. 9, 7 (1976).

${ }^{20} \mathrm{H}$. Lefebvre-Brion, in Molecules in Physics, Chemistry, and Biology, edited by Jean Maruani (Reidel, Dordrecht, Holland, 1988), Vol. 2, p. 257.

${ }^{21}$ W. J. Hunt and W. A. Goddard, Chem. Phys. Lett. 3, 414 (1969).

${ }^{22}$ A. E. Douglas, Can. J. Phys. 52, 318 (1974).

${ }^{23} \mathrm{H}$. van Lonkhuyzen and C. A. de Lange, Mol. Phys. 51, 551 (1984).

${ }^{24}$ R. R. Lucchese, G. Raseev, and V. McKoy, Phys. Rev. A 25, 2572 (1982).

${ }^{25}$ R. R. Lucchese, K. Takatsuka, and V. McKoy, Phys. Rep. 131, 147 (1986).

${ }^{26}$ J. A. Stephens and V. McKoy, J. Chem. Phys. 88, 1737 (1988).

${ }^{27}$ A. Msezane and S. T. Manson, Phys. Rev. Lett. 35, 364 (1975); ibid. 48, 473 (1982); J. Lahiri and S. T. Manson, ibid. 48, 614 (1982).

${ }^{28}$ T. A. Carlson, M. O. Krause, W. A. Svensson, P. Gerard, F. A. Grimm, T. A. Whitley, and B. P. Pullen, Z. Phys. D 2, 309 (1986).

${ }^{29} \mathrm{~A}$ Cooper minimum has recently been identified in Rydberg-Rydberg transitions of NO in double-resonance multiphoton ionization studies. See S. Fredin, D. Gauyacq, M. Horani, C. Jungen, G. Lefevre, and F. Masnou-Seeuws, Mol. Phys. 60, 825 (1987).

${ }^{30}$ J. Lahiri and S. T. Manson, Phys. Rev. A 33, 3151 (1986). 